\section{DGNR-Preisträger 2021}

\section{鼠段DGNR}

Deutsche Gesellschaft für Neuroradiologie e.V.

Im Rahmen der 56. Jahrestagung der Deutschen Gesellschaft für Neuroradiologie - neuroRAD wurden die wissenschaftlichen Preise der DGNR verliehen. Wir gratulieren allen Preisträgern und bedanken uns für die zahlreichen hochkarätigen Einsendungen.

\section{Kurt-Decker-Preis}

Die Deutsche Gesellschaft für Neuroradiologie verleiht den von ihr zum Gedächtnis an Herrn Prof. Dr. Kurt Decker und zur Förderung des Faches Neuroradiologie im deutschsprachigen Gebiet gegründeten Kurt-Decker-Preis 2021 an

\section{PD Dr. med. Dennis M. Hedderich (Technische Universität München)}

als Auszeichnung für herausragende wissenschaftliche Leistungen für die Arbeit

„Langzeiteffekte der Frühgeburt auf das Gehirn: Morphologische Charakterisierung, Pathophysiologie und Lebensspannenperspektive“.

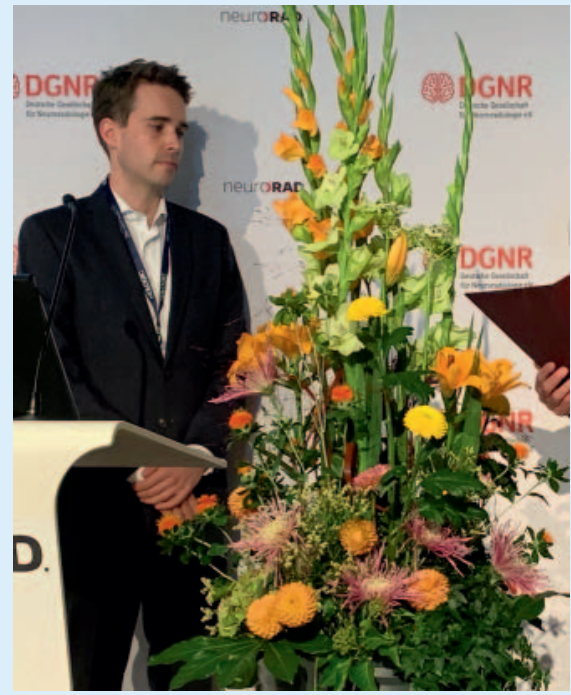

PD Dr. Dennis Hedderich von der TU München bei der Verleihung des Kurt-DeckerPreises 2021. (copyright R. Thäsler/KuKM) 


\section{Marc-Dünzl-Preis}

Die Deutsche Gesellschaft für Neuroradiologie verleiht den von ihr zum Gedenken an Herrn Marc Dünzl und zur Förderung des jungen wissenschaftlichen Nachwuchses ausgeschriebenen Marc-Dünzl-Preis 2021 an

\section{Dr. med. Alexander Rau \\ (Universitätsklinikum Freiburg)}

als Auszeichnung für herausragende wissenschaftliche Leistungen für die Arbeit

„Diffusion microstructure imaging in progressive supranuclear paralysis: Reduced axonal volumes in the superior cerebellar peduncles, dentato-rubro-thalamic tract, ventromedial thalami and frontomesial white matter"

\section{DGNR-Interventionspreis}

Die Deutsche Gesellschaft für Neuroradiologie verleiht den von ihr zur Würdigung innovativer und herausragender Arbeiten auf dem Gebiet der interventionellen Neuroradiologie ausgeschriebenen DGNRInterventionspreis 2021 an

\section{Dr. med. Alexander Kollikowski (Universitätsklinikum Würzburg)}

zur Anerkennung seiner Publikationen

zur Ermöglichung und Standardisierung der Entnahme ischämischer Blutproben unter

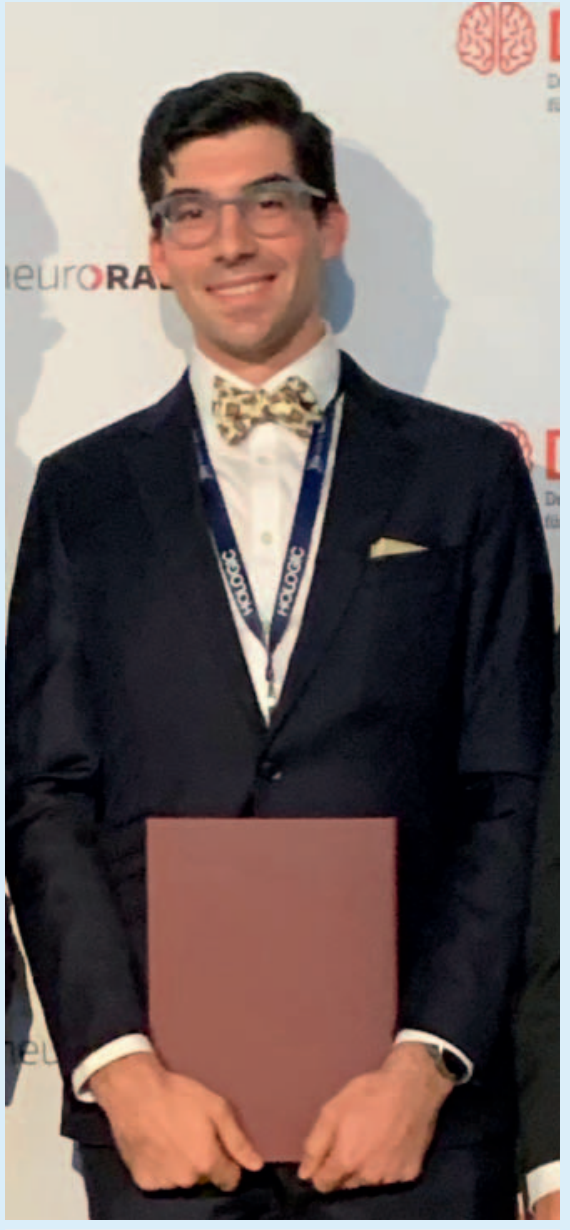

Dr. Alexander Rau vom Universitätsklinikum Freiburg erhielt den Marc-Dünzl-Preis 2021. (copyright: R. Thäsler/KuKM)

Okklusionsbedingung während der mechanischen Thrombektomie.

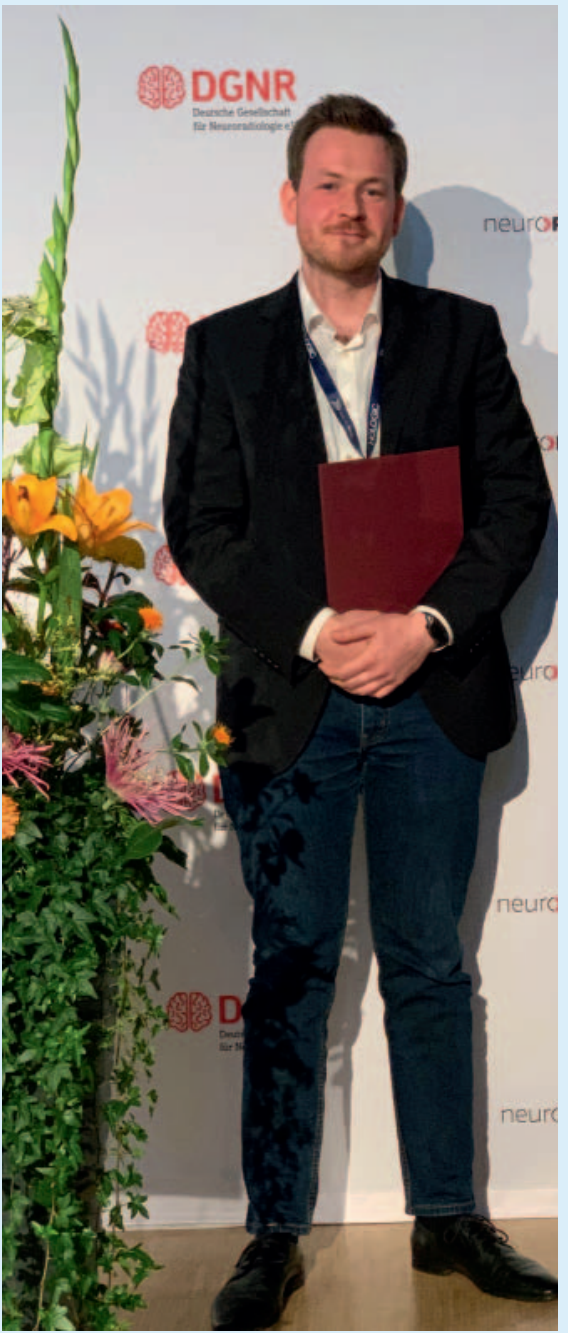

Der DGNR-Interventionspreis ging 2021 an Dr. Alexander Kollikowski vom Universitätsklinikum Würzburg. (copyright: R. Thäsler/ KuKM) 